\title{
CHITOSAN $\mathrm{Fe}_{2} \mathrm{O}_{3}$ NANO COMPOSITES COMPETENT FOR REMOVAL OF HEAVY METAL WATER POLLUTANT'S $\left(\mathrm{Hg}^{+2}, \mathrm{Co}^{+2}, \mathrm{Ni}^{+2}\right)$
}

\author{
Imtiyaz Rasool Parrey* and Hilal UI Zaman \\ Govt. Degree College for Women, Anantnag, Jammu and Kashmir, India \\ *Corresponding author Email: imtiyazchemsitry@gmail.com
}

\begin{abstract}
In this examination, novel chitosan/ $\mathrm{Fe}_{2} \mathrm{O}_{3}$ nanocomposite was incorporated and evaluated as an adsorbent for expelling thorium (IV) particles from the fluid arrangement. The Ch/Fe- nanocomposites were labeled by $x$-ray diffraction (XRD), Brunauer-EmmettTeller (BET) Fourier Transform Infrared Spectroscopy (FT-IR) and Scanning electron microscopy (SEM). The most elevated $\mathrm{Hg}^{2+}$ evacuation yield was seen as 99.3 percent utilizing the 2:1 (w/w) nanocomposite at $\mathrm{pH} 6$ out of $100 \mathrm{ppm}$ of $\mathrm{Hg}^{2+}$ arrangement. The $\mathrm{Ni}$ ${ }^{2+}$ expulsion yield was seen as 98.5 percent utilizing the $2: 1(\mathrm{w} / \mathrm{w})$ nano composite at $\mathrm{pH}$ 5.5 in 100 ppm of $\mathrm{Hg}+2$. The Co2+ expulsion yield was 98.7 percent at pH 5.5 in 60 ppm of $\mathrm{Co}^{2+}$ arrangement utilizing the 4:1 $(\mathrm{w} / \mathrm{w})$ nanocomposite.
\end{abstract}

Key words: Chitosan/Fe2O3, Adsorbent, XRD, FT-IR

\section{Introduction}

Nanotechnology is viewed as a novel innovation that is advancing quickly and spreading its application spaces in all measurements. Nanotechnology incorporates development, assessing, and controlling issue at the nuclear, sub-atomic, macromolecular, and micro molecular scales, with the end goal that the subsequent material has one of a kind and various properties from the parent mass material (Bhatt and Tripathi, 2011; Khan, Mobin, Abbas, AlMutairi, and Siddiqui, 2017). The particles created through this are named as designed nanoparticles (ENPs) or essentially nanoparticles (NPs). ENPs are characterized as particles having, at any rate, one outside measurement somewhere in the range of 1 and 100nm (Shi, Abid, Kennedy, Hristova, and Silk, 2011; Thuesombat, Hannongbua, Akasit, and Chadchawan, 2014). Nanotechnology discovers applications in a broad scope of segments extending from agribusiness, food creation and bundling, clinical symptomatic, pharmaceutical, the nano-based epitome of pesticides, hereditary material conveyance in plants, sedate conveyance in people, and malignant growth treatment to some more (De Oliveira, Campos, Bakshi),

Abhilash \& Fraceto, 2014; Ghormade et al., 2011; Gogos, Knauer, \& Bucheli, 2012). Besides these, its most anticipated applications have been for remediation of environmental compartments like water and soil [1-5]. These fuse treatment of wastewater, cleaning of groundwater, and remediation of soil polluted with harms (Karn, Kuiken, and Otto, 2009; Kuiken, 2010; Rogueda and Traini, 2007; Roh, Park, Park, and Choi, 2010; Shi, Votruba, Farokhzad, and Langer, 2010). Manganese Oxides-Based Nanomaterials Manganese oxides nanoparticles have additionally been accounted for to expel substantial metals from wastewater and Nano sized manganese dioxide just as hydrous manganese oxide $(\mathrm{HMO})$ in which $\mathrm{Mn}$ is quadrivalve are for the most part talked about here. Nano crystalline manganese oxide was found to have a high surface region which adds to its great adsorption execution. Wang et al. blended a novel free weight like manganese dioxide/gelatin and examined its adsorption exhibitions towards $\mathrm{Pb}$ (II) and $\mathrm{Cd}$ (II) [6-8]. The cluster adsorption study demonstrated that the most extreme adsorption limits towards 
$\mathrm{Pb}$ (II) and $\mathrm{Cd}$ (II) were 318.7 and $105.1 \mathrm{mmol} . \mathrm{g}-1$ individually by figuring from the Langmuir model. The adsorption motor followed the pseudo-second-request model. Also, this nanocomposite could be exploited to treat genuine water in the wake of being upheld on an amino-functionalized PMMA plate. High expulsion limit, straightforward activity, and great dependability inferred that this nanocomposite could be applied to rewarding overwhelming metals in genuine water. Moreover, nanoscale manganese dioxide could likewise be utilized in the adsorption and oxidation of $\mathrm{TI}(\mathrm{I})$ in wastewater [8-10]. This group adsorption procedure could be done in $15 \mathrm{~min}$ and the greatest evacuation limit was determined to be $672 \mathrm{mg} . \mathrm{g}-1$ by utilizing the Langmuir model, showing the capability of nanosized manganese oxides to be utilized in TI treatment. What's more to these metals, manganese oxides-based nanomaterials have additionally been accounted for to treat other overwhelming metals like U, $\mathrm{Cd}(\mathrm{II}), \mathrm{Cu}(\mathrm{II}), \mathrm{Pb}$ (II), $\mathrm{Zn}(\mathrm{II}), \mathrm{Hg}$ (II, etc. [11-13]. Hydrous manganese oxide $(\mathrm{HMO})$ is another sort of manganese oxides that have indicated its points of interest in overwhelming metals evacuation thinking about its high surface region, permeable structures, and plenteous destinations for adsorption. The coordination science likewise assumes a noteworthy job in adsorption of HMO since hydroxyl bunches on the HMO surface could facilitate with substantial metal particles [14]. The adsorption of substantial metal particles onto HMO was typically involved in two stages: the snappy adsorption of overwhelming metals onto the outer surface and the moderate intra-molecule dissemination along with the small scale pore dividers of $\mathrm{HMO}$. As of late, wan et al. arranged an $\mathrm{HMO}-\mathrm{BC}$ nanocomposite by impregnating the $\mathrm{HMO}$ nanoparticles into the biochar (BC) [15]. HMO-BC nanocomposite showed a brilliant expulsion impact towards $\mathrm{Pb}$ (II) and $\mathrm{Cd}$ (II) in a wide scope of $\mathrm{pH}$. The mix of $\mathrm{HMO}$ and $\mathrm{BC}$ could maintain a strategic distance from the disadvantages of utilizing BC exclusively, for example, the unacceptable adsorption limit and poor selectivity [16-18]. Fixed-bed segment adsorption tests exhibited that the powerful treatment capacity of HMO-BC for a recreated $\mathrm{Pb}$ (II) or $\mathrm{Cd}$ (II) [19-21] containing wastewater was around 4-6 times higher than that of the BC have. Consequently, HMO-BC is a promising option for evacuating overwhelming Metals from contaminated water. Natural Polymer-Supported Nanocomposites Polymeric hosts have numerous unprecedented properties, for example, magnificent mechanical quality [22-23].

\section{Synthesis of Chitosan $\mathrm{Fe}_{2} \mathrm{O}_{3}$ Nano Composites}

Everything the chosen reagents were of analytical grade and purchased from Merck. The stock solutions for preparation of $\mathrm{Co}^{+2}, \mathrm{Hg}^{+2}, \mathrm{Ni}^{+2}$ were prepared by dissolving respective metal salt in deionized water. The starter $\mathrm{pH}$ of arrangements was estimated by nitric corrosive (HNO3; 99.9 percent) or sodium hydroxide (NaOH; 99.9 percent) arrangements and Metrohm pH meter model 744. Sartorius Electrical Balance Model BP 221S, Nabertherm heater and blender HT Infors AG model CH-4103-BOT were utilized to direct the investigations. Blend of adsorbent was finished by utilizing aqueous reactor, which was structured and made of treated steel $316 \mathrm{~L}$ just as traditional autoclaves with Teflon liner by volume of $100 \mathrm{~mL}$.

Amalgamation of $\mathrm{Fe}_{2} \mathrm{O}_{3}$ Nano molecule (Fe-ONPs) Fe-ONPs were set up by aqueous combination strategy. One gram of $\mathrm{FeCl}_{3} .6 \mathrm{H} 2 \mathrm{O}, 3.5 \mathrm{~g}$ of hexa-methylene diamine and $1.5 \mathrm{~g}$ of trisodium citrate were broken down in $100 \mathrm{~mL}$ of refined water at a temperature of $70 \mathrm{C}$ and blended for $1 \mathrm{~h}$. The arrangement was filled aqueous reactor and put in aqueous autoclave at $200 \mathrm{C}$ for $10 \mathrm{~h}$. The delivered Fe-ONPs were washed with deionized water and dried at room temperature. A blend of water and ethanol $(1: 5(\mathrm{v} / \mathrm{v}))$ was added dropwise to the suspensions of FeONPs under incredible attractive mixing and left to make due with $1 \mathrm{~h}$. The altered Fe-ONPs was washed with refined water a few times and dried in the stove at $110 \mathrm{C}$ for $3 \mathrm{~h}$.

Combination of $\mathrm{Ch} / \mathrm{Fe}-\mathrm{Onc} \mathrm{Ch}$ medium atomic load with the level of deacetylation somewhere in the range of 75 and 85 percent was utilized so as to get ready $\mathrm{Ch} / \mathrm{Fe}-\mathrm{Onc}$. Ch $(1.67 \mathrm{~g})$ was broke up in $50 \mathrm{~mL}$ of $0.1 \mathrm{~N}$ hydrochloric corrosive arrangement and blended for $1 \mathrm{~h}$. At that point $0.1 \mathrm{~g}$ of arranged Fe-ONPs was completely scattered in the 
Ch arrangement followed by the expansion of hydrochloric corrosive arrangement. Sampler was utilized to trickle out the subsequent arrangement into $0.1 \mathrm{~N}$ sodium hydroxide arrangements. Beads were shaped as circular Nano composites with a breadth of $0.45 \mathrm{~cm}$. The subsequent $\mathrm{Ch} / \mathrm{Fe}-\mathrm{Onc}$ was kept up in sodium hydroxide answer for $24 \mathrm{~h}$ and, at last, washed with deionized water and dried at room temperature. Subjective synthetic structure assessment was done by means of Fourier change infrared (FTIR) investigation (Vector22 Brucker Company, USA). Th4p investigation was followed up by utilizing a Perkin-Elmer Optima 2000 DV Model Inductively Coupled Plasma-Optic Emission Spectrometry (ICPOES)

\section{$X$ ray Diffraction}

The XRD patterns of $\mathrm{Ch} / \mathrm{Fe}_{2} \mathrm{O}_{3}$ nanocomposites and its base materials $\left(\mathrm{Ch} / \mathrm{Fe}_{2} \mathrm{O}_{3}\right)$ were recorded using a Rigaku Ultima IV diffractometer with $\mathrm{Hg}^{+2} \mathrm{Ka}(\lambda=0.2189 \mathrm{~nm})$ radiation. The diffraction intensities were recorded from 5 to $40^{\circ}(2 \theta)$ angle. The $d$ spacing of MMT layers was calculated using the Bragg equation

Figure 01: XRD patterns of (a) Fe- Ch nono composite (b) Fe- Ch nanocomposite (1:1), (c) Fe- Ch nonocomposite (2:1), (d) Fe- Ch noncomposite (4:1)

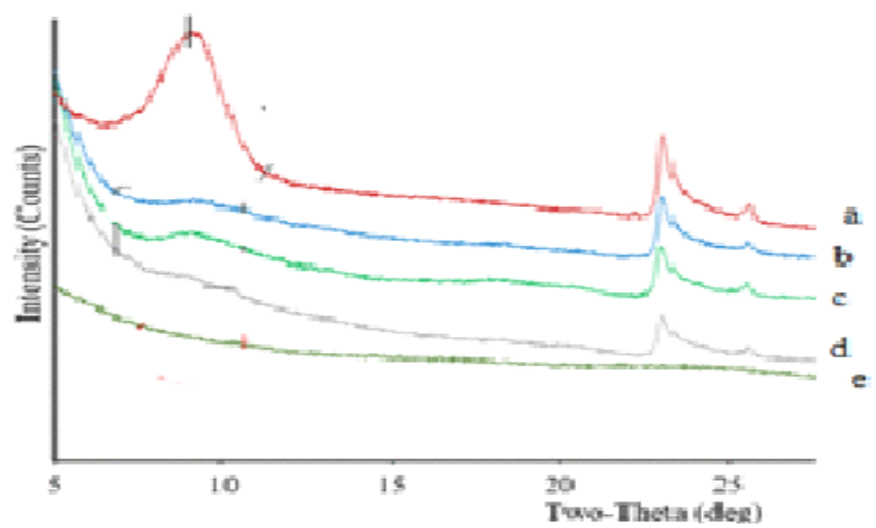

\section{SEM Analysis}

SEM analyses of Nano composite samples were carried out on a Sigma HD Zeuss scanning electron microscope at an acceleration voltage of $20 \mathrm{kV}$ (SE detector). The main objective of this characterization technique is the identification of materials morphology that represents a key factor to analyze in the adsorption process.

Figure 02: SEM micrographs of (a) Fe- Ch nanocomposite (b) Fe- Ch nanocomposites (c) Fe- Ch nanocomposites (d) Fe- Ch nanocomposites
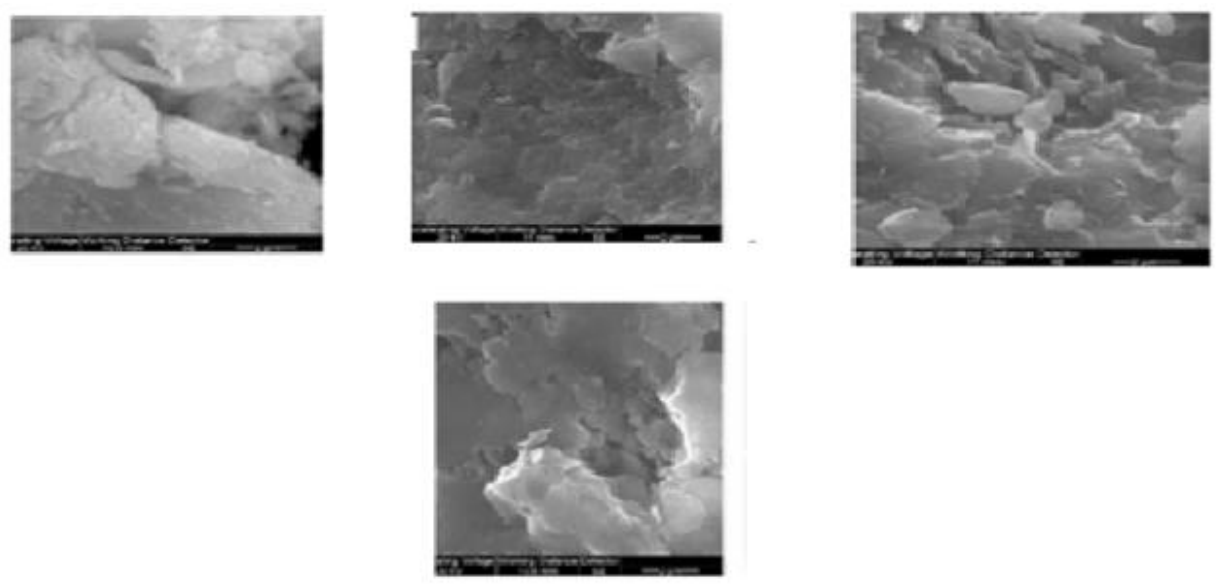


\section{Adsorption Study Report \\ Adsorption Isotherms Conclusion}

Adsorption is an active process that is usually defined by isotherms, determined by fitting mathematical models to experimental data. The information provided by these models quantifies how the adsorbate is distributed between the solid and liquid phases. In this investigation, the Langmuir, eq 1, and the Freundlich, isotherms were used to study the equilibrium adsorption of $\mathrm{Hg}^{+2}(1: 1), \mathrm{Co}^{2+}(2: 1)$ and $\mathrm{Ni} 2+$ ions onto $\mathrm{Ch} / \mathrm{Fe}_{2} \mathrm{O}_{3}$ nanocomposites $(4: 1 \mathrm{w} / \mathrm{w})$. The suitability of both models was quantified with the correlation factor $\left(R^{2}\right)$ obtained from linear fitting and adsorption capacity constant (qe).

$\ln \mathrm{q}_{\mathrm{e}}=\ln \mathrm{K}_{1}+1 / \mathrm{n} \ln \mathrm{Ce}(2)$

In eqs 1 and $2 q_{\mathrm{m}}$ is the amount of heavy metal present in a monolayer $(\mathrm{mg} / \mathrm{g}), C_{\mathrm{e}}$ is the equilibrium concentration of heavy metal remaining in the solution $(\mathrm{mg} / \mathrm{L}), q_{\mathrm{e}}$ is the quantity of the heavy metal adsorbed by per unit of adsorbent at equilibrium $(\mathrm{mg} / \mathrm{g})$,

\section{Absorption Studies Isothermal Study}

Comparing the correlation coefficients $(R 2)$ obtained by fitting the adsorption results with the Langmuir and Freundlich isotherms for $\mathrm{Hg}^{+2}, \mathrm{Co}^{2+}$ and $\mathrm{Ni}^{2+}$, the Langmuir isotherm provided a better description of the data than the Freundlich isotherm for $\mathrm{pH}$ values of 5 , 5.5 and 6 , but when the $\mathrm{pH}$ was 3 , the Freundlich isotherm outperformed the Langmuir isotherm. This suggests that a monolayer adsorption process on homogeneous sites (described by the Langmuir isotherm) takes place in aqueous solutions with lower acidity. At lower $\mathrm{pH}$ esteems, the Langmuir model may go amiss from the genuine adsorption conduct because of the presumption of no cooperations between sorbate particles. By the by, this model gives palatable understandings of a wide number of tests that predominantly present chemisorption. Then again, the Freundlich isotherm models multilayer adsorption forms on heterogeneous surfaces, and its better fit recommend that this component depicts the procedure at $\mathrm{pH} 3$ all the more precisely.

Figure 03: Substantial metal adsorption on Nano composite at pH values of $\mathrm{Ch} / \mathrm{Fe}_{2} \mathrm{O}_{3}(1: 1)$ and $\mathrm{Ni}^{2+}$ ions, (b) (2:1) and $\mathrm{Co}^{2+}$ ions, (c) $\mathrm{Ch} / \mathrm{Fe}_{2} \mathrm{O}_{3}(4: 1)$ and $\mathrm{Hg}^{2+}$ ions,

(d) $\mathrm{Ch} / \mathrm{Fe}_{2} \mathrm{O}_{3}(1: 1)$.
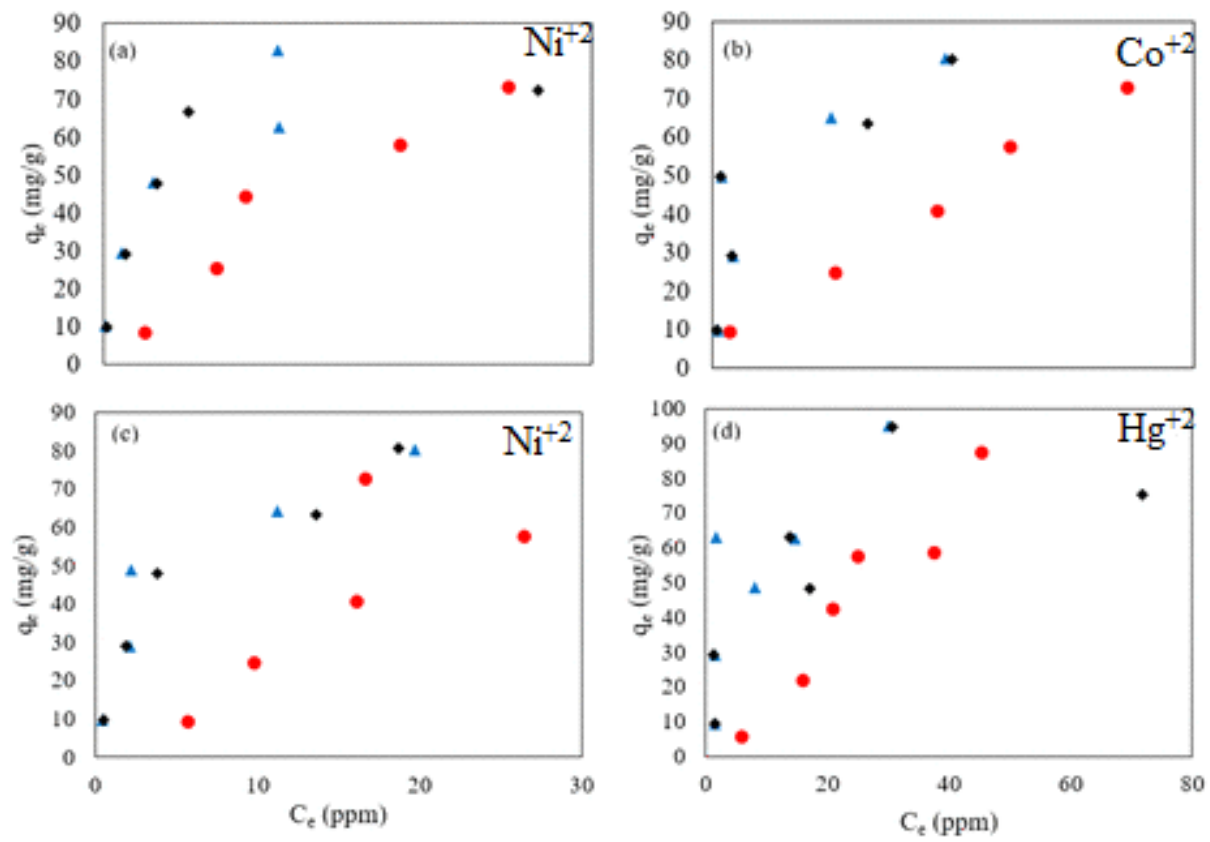
Figure 04: $\mathbf{R}_{\mathrm{L}}$ dimensionless factor versus initial concentration for (a) $\mathrm{Hg}^{2+}$ ions and (b) $\mathrm{Ni}^{2+}$ ions and (c) $\mathrm{Co}^{2+}$ at $\mathrm{pH}$ estimations of 5 and 6 with the $\mathrm{Ch} / \mathrm{Fe}_{2} \mathrm{O}_{3}$
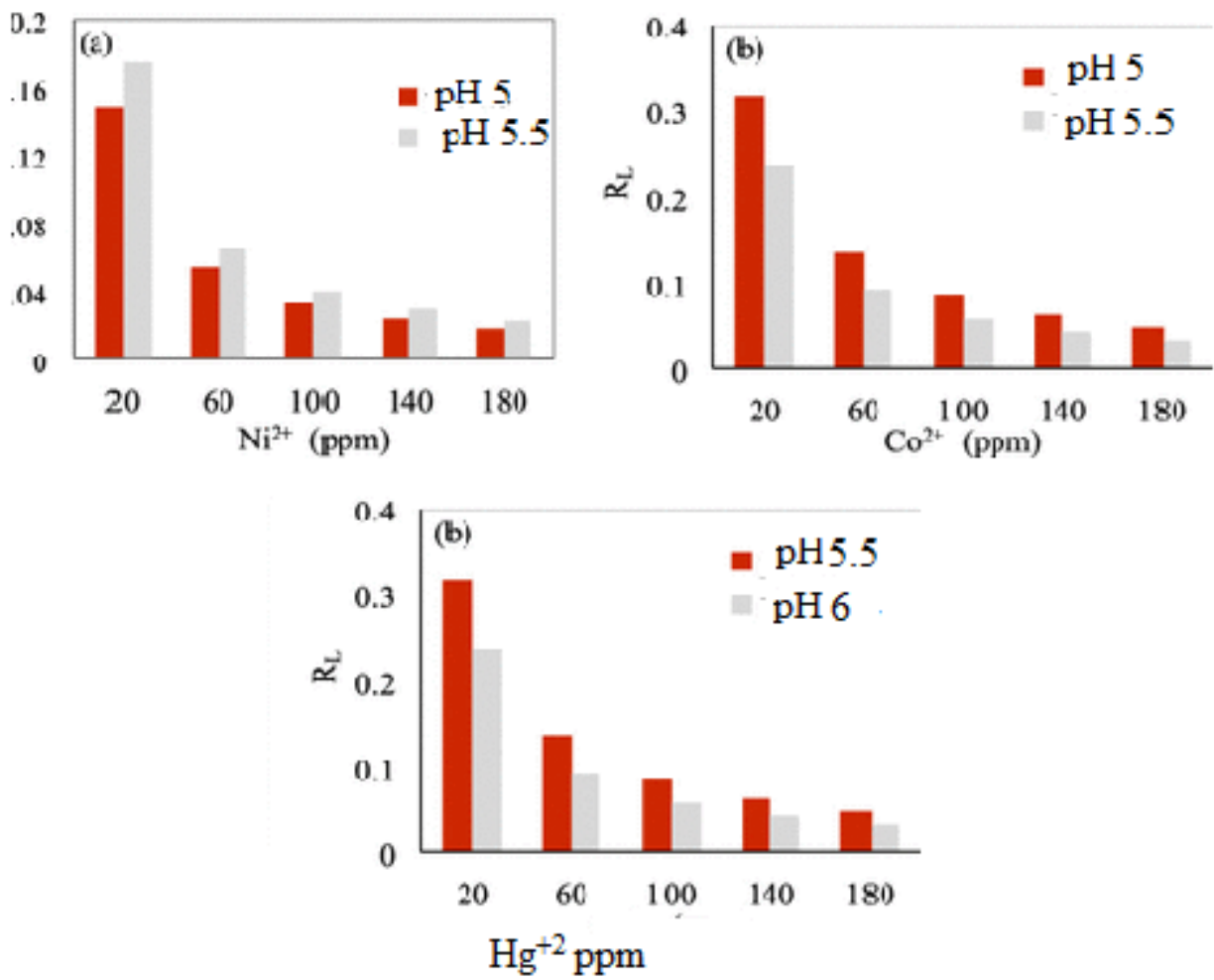

\section{Conclusion}

This exploration scheme assessed the adsorption limit of nanocomposites dependent on $\mathrm{Ch} / \mathrm{Fe}_{2} \mathrm{O}_{3}$ for the expulsion of overwhelming metal particles $\left(\mathrm{Hg}^{2+}, \mathrm{Ni}^{2+}\right.$ and $\left.\mathrm{Co}^{2+}\right)$ from wastewater. SEM and XRD to evaluate utilitarian gatherings, morphologies, and structures described the readied composites. Adsorption of $\mathrm{Hg}^{2+}, \mathrm{Ni}^{2+}$ and $\mathrm{Co}^{2+}$ particles is best depicted by the Langmuir isotherm at higher $\mathrm{pH}$ esteems, as prove by a coefficient of relationship near one.

\section{References}

1. Abdullah, J.A., Lafi, A.G.A., Amin, Y., Alnama, T. A. (2013). Styrofoam-nano manganese oxide based composite:168. Mukherjee, J.; Ramkumar, J.; Shukla, R.; Tyagi, A.K. Sorption characteristics of nano manganese oxide: Efficient sorbent for removal of metal ions from aqueous streams. J. Radioanal. Nuclear Chem, 297, 49-57

2. Abdullah, J.A., Lafi, A.G.A.; Amin, Y.; Alnama, T. A. (2018). Styrofoam-nano manganese oxide based composite: Preparation and application for the treatment of wastewater. Appl. Radiat. Isotopes, 136, 73.

3. Anandan, S., Kathiravan, K., Murugesan, V., Ikuma, Y. (2009). Anionic IO3- non-metal doped $\mathrm{TiO} 2$ nanoparticles for the photocatalytic degradation of hazardous pollutant in water. Catal. Commun. 10, 1014-1019.

4. Byungryul, A., Steinwinder, T.R., Dongye, Z. (2017). Selective removal of arsenate from drinking water using apolymeric ligand exchanger. Water Res. 2005, 39, 49935004.166. Huo, L.; Zeng, X.; Su, S.; Bai, L.; Wang, Y. Enhanced removal of as (V) from aqueous solution using modified hydrous ferric oxide nanoparticles. Sci. Rep., 7, 40765.

5. Fan, M., Boonfueng, T.; Xu, Y.; Axe, L., Tyson, T.A. (2005). Modeling Pb sorption to microporous amorphous oxides as discrete particles and coatings. J. Colloid Interface Sci., 281, 39-48

6. Ghiloufi, I., Mir, L.E. (2015). Preparation and characterization of nanoporous resin for heavy metal removal from aqueous solution. J. Water Supply Res. Technol.-Aqua, 64, 316-325. 
7. Hadadian, M., Goharshadi, E.K., Fard, M.M., Ahmadzadeh, H. (2018). Synergistic effect of graphene nanosheets and zinc oxide nanoparticles for effective adsorption of $\mathrm{Ni}(\mathrm{II})$ ions from aqueous solutions. Appl. Phys. A, 124, 239.

8. Huangfu, X., Jin, J., Lu, X., Wang, Y., Liu, Y., Pang, S.Y., Cheng, H., Xiang, Z., Ma, J. (2015). Adsorption and Oxidation of Thallium(I) by a Nanosized Manganese Dioxide. Water Air Soil Pollut. 226, 2272.

9. Huangfu, X., Jin, J., Lu, X., Wang, Y, Liu, Y., Pang, S.Y., Cheng, H., Xiang, Z., Ma, J. (2015). Adsorption and Oxidationof Thallium(I) by a Nano sized Manganese Dioxide. Water Air Soil Pollute.226, 2272.

10. Hui, Q., Shujuan, Z., Bingcai, P., Weiming, Z., Lu, L. (2012). Effect of sulfate on Cu(II) sorption to polymer-supported nano-iron oxides: Behavior and XPS study. J. Colloid Interface Sci. 2012, 366, 37-43.162.

11. Singh, S.K., Subramanian, V., Gibbs, R.J. (1984). Hydrous FE and MN oxidesScavengers of heavy metals in the aquatic environment. Crit. Rev. Environ. Control, 14, 33-90.

12. Hui, Q., Zhang, S., Pan, B., Zhang, W., Lu, L. (2013). Oxalate-promoted dissolution of hydrous ferric oxide immobilized within nano porous polymers: Effect of ionic strength and visible light irradiation. Chem. Eng. J., 232, 167-173.

13. Kumar, K.Y., Muralidhara, H.B., Nayaka, Y.A., Balasubramanyam, J., Hanumanthappa, $\mathrm{H}$. (2013). Low-cost synthesis of metal oxide nanoparticles and their application in adsorption of commercial dye and heavy metal ion in aqueous solution. Powder Technol., 246, 125-136.

14. Lisha, K.P.; Maliyekkal, S.M.; Pradeep, T. (2010). Manganese dioxide nano whiskers: A potential adsorbent for the removal of $\mathrm{Hg}(\mathrm{II})$ from water. Chem. Eng. J., 160, 432439.

15. Lockwood, R.A., Chen, K.Y. (1973). Adsorption of mercury(II) by hydrous manganese oxides. Environ. Sci. Technol, 7, 1028-1034.

16. Mukherjee, J., Ramkumar, J., Shukla, R., Tyagi, A.K. (2013). Sorption characteristics of nano manganese oxide: Efficient sorbent for removal of metal ions from aqueous streams. J. Radioanal. Nuclear Chem, 297,49-57.

17. Rafiq, Z., Nazir, R., Durr-e-Shahwar; Shah, M.R., Ali, S. (2014). Utilization of magnesium and zinc oxide nano-adsorbents as potential materials for treatment of copper electroplating industry wastewater. J. Environ. Chem. Eng., 2, 642-651.

18. Sheela, T., Nayaka, Y.A., Viswanatha, R., Basavanna, S.. Venkatesha, T.G. (2012). Kinetics and thermodynamics studies on the adsorption of $\mathrm{Zn}(\mathrm{II}), \mathrm{Cd}(\mathrm{II})$ and $\mathrm{Hg}$ (II) from aqueous solution using zinc oxide nanoparticles. Powder Technol, 217, 163-170.

19. Somu, P.; Paul, S. (2018). Casein based biogenic-synthesized zinc oxide nanoparticles simultaneously decontaminate heavy metals, dyes, and pathogenic microbes: A rational strategy for wastewater treatment. J. Chem. Technol. Biotechnol.

20. Sposito, G.(1983). On the surface complexation model of the oxide-aqueous solution interface. J. Colloid Interface Sci., 91, 329-340.

21. Wan, S., Wu, J., Zhou, S., Rui, W., Gao, B.,Feng, H. (2018). Enhanced lead and cadmium removal using biochar-supported hydrated manganese oxide (HMO) nanoparticles: Behavior and mechanism. Sci. Total Environ, 616-617, 1298-1306.

22. Wang, X., Huang, K., Chen, Y., Liu, J., Chen, S., Cao, J., Mei, S., Zhou, Y., Jing, T. (2018). Preparation of dumbbell manganese dioxide/gelatin composites and their application in the removal of lead and cadmium ions. J. Hazard. Mater, 350, 46-54.

23. Wang, X. Huang, K., Chen, Y., Liu, J.; Chen, S., Cao, J., Mei, S.; Zhou, Y., Jing, T. (2018). Preparation of dumbbell manganese dioxide/gelatin composites and their application in the removal of lead and cadmium ions. J. Hazard. Mater.350, 46-54

24. Zhang, Y. Li, Z. (2017). Heavy metals removal using hydrogel-supported nanosized hydrous ferric oxide: Synthesis, characterization, and mechanism. Sci. Total Environ., 580, 776-786. 